\author{
Anne Verjus \\ GAPP-CNRS \\ Ecole Normale Supérieure de \\ Cachan \\ Bâtiment Laplace \\ 61, Avenue du Président Wilson \\ 94235 Cachan cedex
}

Email : verjus@gapp.ens-cachan.fr

\title{
Entre principes et pragmatisme : députés et sénateurs dans les premiers débats sur le suffrage des femmes en France (1919-1922)
}

Politix, automne 2000

\author{
Gapp-CNRS \\ ENS de Cachan \\ 61, avenue du Président Wilson \\ 94235 Cachan Cedex
}


Résumé :

L'échec du projet de droit de suffrage des femmes en 1922 ne doit pas cacher le succès qu'il a d'abord rencontré à la Chambre des députés, en 1919. Pourtant, malgré la contradiction entre les deux issues des scrutins, une même rhétorique "sexualiste" est à l'œuvre dans les deux assemblées, à savoir : une vision des femmes comme classe de sexe, au comportement politique distinct de celui des hommes. Si les réponses différent, à seulement trois ans d'intervalles et dans des chambres de composition politique équivalente, c'est pour des raisons à la fois institutionnelles et contextuelles. L'intérêt de ce débat sur le suffrage des femmes est donc non seulement d'apporter des nuances à l'histoire de la résistance toute française au féminisme, mais également de montrer comment une même rhétorique peut rencontrer, selon la Chambre (et donc le degré de responsabilité des parlementaires concernés) où elle est déployée, des réponses parfaitement contradictoires.

Au mois de mai 1919, par un vote massif de 344 voix contre 97, la Chambre majoritairement composée de députés de la gauche accorde aux femmes le droit de voter et d'être élues, ceci dans toutes les élections. Dès le lendemain, Emile Combes, président du groupe de la Gauche démocratique au Sénat, donne pour consigne de rejeter la proposition de loi ; le mot d'ordre s'avère efficace puisque trois ans plus tard la plupart des membres de ce groupe parviennent à empêcher le passage à la discussion des articles. A partir de cette date et pour tout l'entre-deux-guerres, cette proposition en faveur d'un suffrage féminin ne rencontrera plus que l'hostilité de la "Chambre haute".

Qu'est-ce qui a déterminé le refus sénatorial alors que les députés s'étaient montrés, trois ans plus tôt, si unanimement favorables au projet de loi ? La question, loin d'avoir été négligée par l'historiographie, a donné lieu à de multiples interprétations. Mais celles-ci s'étant essentiellement focalisées sur le refus fracassant et pour longtemps définitif du Sénat, elles n'ont que peu pris en compte la contradiction qui, à trois ans d'intervalle, oppose les parlementaires des deux Chambres. Or, le phénomène méritait d'être étudié, ne seraitce que parce qu'il permet de redonner aux contraintes politiques et institutionnelles du moment toute leur force de détermination dans l'issue des débats ; en outre, par la comparaison qu'il nécessite entre les débats de 1919 et 1922, il met à jour des catégories de pensée qui ne sont pas celles qu'on attendait. 
Trois types d'interprétations se partagent actuellement le champ historiographique sur la question du retard français en matière de droit de vote des femmes : celle de Steven Hause et Anne Kenney, selon lesquels la France s'est opposée au droit de vote des femmes en raison d'une pensée politique globalement anti-individualiste ${ }^{1}$; celle de Pierre Rosanvallon pour qui c'est au contraire la philosophie politique individualiste du droit public français qui a empêché l'intégration des femmes au motif qu'elles n'étaient pas considérées comme des individus (et ce, contrairement aux pays anglo-saxons qui ont intégré les femmes en tant que douées de vertus spécifiques à leur sexe) ${ }^{2}$; celle enfin de l'Histoire des femmes qui fait de la lutte entre les sexes le principal élément explicatif de l'attitude des parlementaires à l'égard (du suffrage) des femmes ${ }^{3}$.

Malgré leurs conclusions divergentes, ces trois types d'interprétations ont un point commun, qui est de négliger le vote de la Chambre en 1919 , en focalisant leur recherche sur celui de 1922. On peut voir là un effet pervers du métier d'historien qui, par sa connaissance des suites données à ce vote, préfère mettre l'accent sur des événements auxquels l'Histoire a donné raison au détriment d'autres devenus caduques et dont la signification est, par force, dévaluée. Mais le souci d'introduire de la cohérence, propre à toute tentative d'interprétation, est peut-être également en cause : car pour comprendre la contradiction entre les votes de 1919 et 1922, sachant que ce sont les élus du même parti qui dans les deux cas ont emporté la décision, on a paradoxalement misé sur la cohérence des parlementaires pour en déduire que le vote de 1919 était cynique et insincère. Or, le postulat de l'unité du Parlement mérite d'être interrogé : certes on peut supposer que les membres de l'élite politique partagent plus ou moins les mêmes représentations ; pour autant, ce n'est pas forcément cette représentation qui guide le vote des députés et des sénateurs. On peut même faire l'hypothèse que c'est tout le contraire puisque les deux votes sont, de fait, en contradiction. Autrement dit, ce que nous nous proposons de montrer, c'est qu'au-delà de leurs représentations communes (par définition), on peut aussi comprendre les positions contradictoires des uns et des autres en se fondant sur ce qui les distingue plutôt que sur ce qui les rapproche, à savoir : leur situation institutionnelle de députés ou de sénateurs.

Pour ce faire, une comparaison entre les discours de 1922 et 1919 s'avère nécessaire : on constatera en premier lieu que ce ne sont pas les "mentalités" qui sont en cause dans le rejet du projet puisqu'en 1922, opposants et partisans partagent la même vision (sexualiste) du corps électoral ; ceci d'autant moins qu'en 1919, le principe de la lutte entre les sexes avait au contraire permis d'emporter l'adhésion enthousiaste des députés en faveur du suffrage féminin. Au-delà de l'idéologie commune aux hommes politiques de cette période, ce qui détermine leurs votes respectifs en 1922 et 1919 et permet d'expliquer le rejet du suffrage féminin pour tout l'entre-deux-guerres, c'est leur appartenance à l'une ou l'autre Chambre ; autrement dit, la mesure à la fois de leurs fonctions électives selon qu'ils sont députés ou sénateurs,

\footnotetext{
${ }^{1}$ Cf. Steven C. Hause and Anne R. Kenney, Women's Suffrage and Social Politics in the French Third Republic, Princeton University Press, 1984.

${ }^{2}$ Cf. Pierre Rosanvallon, Le sacre du citoyen. Histoire du suffrage universel en France, Paris, Gallimard, 1992.

${ }^{3}$ Un bon aperçu des problématiques de ce champ disciplinaire dans l'ouvrage collectif dirigé par Christine Bard, d'antiféminisme, Paris, Fayard, 1999. 
des rapports que les deux chambres entretiennent entre elles et enfin, du degré de responsabilité qu'induit leur positionnement dans le processus législatif.

\section{Le sexualisme, fond commun de la pensée politique}

En novembre 1922, les sénateurs débattent longuement de la question du droit de suffrage féminin sur la base du texte voté par la Chambre trois années auparavant. L'issue des débats, c'est-à-dire le rejet de la discussion des articles, n'est une surprise pour personne. On savait depuis 1919 que les sénateurs, ou plus exactement ceux de la Gauche démocratique - groupe parlementaire le plus important du Sénat ${ }^{4}-$ s'opposeraient à toute participation électorale des femmes. Mais le débat n'en est pas moins d'un grand intérêt quoique, selon toute vraisemblance, joué d'avance - ne serait-ce que parce que les positions défendues ne suivent pas nécessairement l'appartenance partisane des uns et des autres. C'est le cas notamment de ces sénateurs de la gauche démocratique qui défendent le droit de suffrage féminin contre leur propre groupe et contre la Commission dont le rapporteur, Alexandre Bérard, et le président, Régismanset, sont aussi deux membres éminents ${ }^{5}$. C'est que la discipline de parti n'est toujours pas en usage au lendemain de la guerre - et au sein de la gauche démocratique encore moins qu'ailleurs ${ }^{6}$; aussi les quelques figures qui dans ce groupe défendent la cause des femmes, comme Louis Martin ou Fernand Merlin, peuvent-elles espérer un renversement de la tendance en faveur du projet voté par les députés. Au terme du débat, la majorité du refus s'avère assez étroite d'ailleurs, puisque seulement 22 voix font la différence. La cause aurait donc pu être gagnée. Si elle ne le fut pas c'est qu'en 1922 et sur la question du suffrage féminin, on observe en fait un commencement de discipline de parti : plus de $60 \%$ des membres de la gauche votent contre la discussion des articles, alors que $70 \%$ des conservateurs votent pour. L'enjeu est donc de taille pour s'imposer ainsi au-delà des objectifs individuels qui priment

\footnotetext{
${ }^{4}$ En 1922, le Sénat se compose à 51,4\% de "progressistes", inégalement répartis entre 4 groupes : 5 socialistes, 141 de la Gauche démocratique, 7 de l'Union démocratique et radicale, 1 de l'union progressiste ; enfin, 5, sans appartenance précise, peuvent être considérés comme proches de la gauche démocratique ; il se compose également, à $39 \%$, de 121 "conservateurs", répartis entre l'Union républicaine et leurs proches $(69+10)$, la Droite (13), et la Gauche républicaine (26) ; on trouve également un républicain démocrate et deux indépendants. Enfin, 29 sénateurs ont une appartenance indéterminée. Statistique établie à partir de Jean Jolly, Dictionnaire des parlementaires français, notices biographiques sur les ministres, sénateurs et députés français de 1889 à 1940, Paris, PUF, 1960-1977.

5 Jacques Régismanset, président de la Commission sur le suffrage des femmes, est sénateur de la Seine et Marne depuis 1891 ; vice président du groupe de la gauche démocratique, et du Sénat à partir de 1917, il a été rapporteur du projet de loi relatif à la liquidation des congrégations. Quant à Alexandre Bérard, rapporteur de la commission, il est une figure importante de la gauche radicale ; il a voté pour ses lois les plus progressistes, dont l'éligibilité des femmes aux conseils des prud'hommes ; ancien vice-président du groupe de la gauche démocratique à la Chambre des députés, devenu vice-président du Sénat, c'est lui qui s'est imposé comme le continuateur de la politique d'obstruction systématique au projet de suffrage des femmes engagée par Emile Combes au lendemain du vote favorable de la Chambre en mai 1919. Tous deux, évidemment, votent contre la discussion des articles en 1922.

${ }^{6}$ Cf. Nicolas Rousselier, "Phénomène de majorité et relation de majorité. Validation limitée d'outils empiriques à propos du résistible déclin de la maison radicale en France", in Politix, no 20, 4ème trimestre 1992, pp. 63-71. ; ainsi que Nicolas Rousselier, Le Parlement de l'éloquence, Paris, Presses de la FNSP, 1997 ; pour une perspective plus globale sur la période, cf. Philippe Bernard, La fin d'un monde. 1914-1929. Nouvelles histoire de la France contemporaine, Vol. 12 Paris, Ed. du Seuil, 1975 , en particulier p. 226.
} 
habituellement ${ }^{7}$. Cet enjeu capable de fédérer les plus indisciplinés n'est rien moins que la "sauvegarde" de la République.

\subsection{Un vote féminin prématuré, inutile et dangereux(justification)}

Pour justifier leur refus, partiel ou radical, du projet voté par la Chambre des députés, les sénateurs axent leurs discours sur trois caractéristiques supposées du vote féminin qui serait prématuré, inutile, et surtout, surnuméraire.

Il est trop tôt. Les femmes ne sont pas assez instruites et manquent trop d'expérience pour ajouter à l'inexpérience des hommes, "qu'une si longue pratique du suffrage n'a pu corriger" ${ }^{8}$, leur propre inexpérience. Ces deux arguments, souvent combinés avec celui du désintérêt tout féminin pour la politique, reviennent régulièrement dans le débat. Ils peuvent étonner par leur mauvaise foi : comment reprocher aux femmes de ne pouvoir être ce qu'elles n'ont jamais été ? En outre, ne sont-ce pas les républicains eux-mêmes qui ont mis en place une instruction destinée à transformer les femmes en républicaines ? Il semble importer peu pour l'heure qu'un bilan si piteux puisse leur être imputé : les sénateurs de la gauche démocratique partagent, du moins lorsqu'ils s'expriment en public, une vision des femmes que résume bien ce passage dans lequel le président de la commission, Régismanset, cite un article de journal :

Régismanset : "Jamais, au grand jamais, continue l'auteur de cet article, les femmes ne discutent la politique du bloc national, elles n'émettent d'opinion quelconque sur les idées de M. Painlevé ou sur la liberté des détroits. Incontestablement elles se moquent de toutes ces vétilles comme de leur première combinaison." (...) "La mode, voilà la grande affaire", ajoute l'auteur en terminant."

Gaudin de Villaine : C'est un vieux garçon qui a dî écrire cela.

M. le président de la commission :Je ne généralise pas. Quand il s'agit d'articles humoristiques, évidemment ce n'est pas l'ensemble de la nation féminine qui est atteinte : tout de même on peut dire que bon nombre de femmes ressemblent au portrait qui vient d'être fait."

Le vote est prématuré ; il est aussi inutile, dans la mesure où les femmes ne le demandent pas. Pourquoi aller au-devant de leur inappétence pour les choses publiques, pourquoi les "forcer" finalement à devenir ce qu'elles ne désirent pas ? L'argument vaut surtout, semble-t-il, pour celles qui aux yeux des sénateurs figurent les "vraies" femmes, ces femmes moyennes qui n'ont pas été touchées par

\footnotetext{
${ }^{7}$ Robert de Jouvenel disait qu'un groupe, "ce n' est pas une organisation politique, c'est une union corporative qui n'a pas été créée pour faire triompher une doctrine, mais pour permettre à un certain nombre d'individus d'arriver sans se bousculer". Robert de Jouvenel, cité par Yves-Henri Gaudemet, Les juristes et la vie politique de la IIIème république, Paris, PUF, 1970 , p. 38. A ne pas confondre avec Henry de Jouvenel, sénateur de la Corrèze qui dans le débat de 1922 vote pour la discussion des articles.

${ }^{8}$ Jean Philip, intervention au Sénat dans la séance du 16 novembre 1922. Sénateur du Gers de 1920 à 1941, membre du groupe de la gauche démocratique, en 1922 il vote pour la discussion des articles.

9 Jacques Régismanet, intervention au Sénat dans la séance du 21 novembre 1922. Adrien Gaudin de Villaine, sénateur de la Manche de 1906 à 1930, adversaire acharné de la République, il siège à droite. En 1922, il vote pour la discussion des articles.
} 
le mouvement féministe, qu'elles soient ces "ménagères" qui se "soucient peu d'aller au scrutin"10 ou ces "cultivatrices de robuste bon sens" qui se "défendent avec quelque peine d'un certain sourire ironique lorsqu'elles apprennent que les sénateurs consacrent tant de temps à décider s'ils doivent leur octroyer ou non un cadeau qu'elles ne sollicitent pas." (Très bien ! très bien ! et applaudissements.) ${ }^{11}$.

$\mathrm{Au}$ fil des débats se dessine le portrait de la future citoyenne selon les sénateurs de la gauche démocratique : ignare, superficielle, éloignée de la politique de son propre fait et satisfaite de sa situation. Comment et pourquoi intégrer une telle masse à un corps électoral qui risquerait de ne pas se remettre d'une telle adjonction ? Car il est un ultime argument qui, joint aux deux autres, achève de faire surgir le spectre du bouleversement : c'est que les femmes, depuis la guerre, sont plus nombreuses que les hommes. Or, si l'on postule comme le font les sénateurs qu'hommes et femmes ont, sur la base de leur spécificité sexuelle, des comportements électoraux distincts, il va de soi que la supériorité numérique des femmes ne pourra qu'entraîner leur suprématie politique. Ce qui est proprement intolérable, comme le fait comprendre le rapporteur de la Commission, Alexandre Bérard :

"La guerre a déséquilibré notre pays et actuellement, le nombre des femmes est supérieur de 1700000 à 1800000 à celui des hommes. Allez-vous décider que, d'un seul coup, les destinées du pays sont remises à ce groupement de femmes, à ces 1800000 femmes qui pourraient ainsi anéantir le vote masculin ? ${ }^{\prime \prime 2}$

L'argument révèle l'emprise de l'idéologie sexualiste ${ }^{13}$ sur les débats ; car il n'y a pas seulement un vote féminin d'une part, et d'autre part ce que l'on pourrait considérer comme le vote universel et neutre exercé par les citoyens. Il y a bel et bien partage du corps électoral en deux moitiés selon l'appartenance sexuelle des uns et des autres. Et le vote du citoyen est un vote masculin que, non contents d'exercer, les hommes ont bien l'intention de conserver comme tel; ce qui nécessite qu'à aucun moment l'"équilibre" démographique entre les deux moitiés, si tant est qu'on laisse à la deuxième l'usage de son droit de vote, ne se fasse à leur détriment. Même un partisan du suffrage féminin comme Fernand Merlin admet qu'til ne saurait être question de voter une loi qui sacrifierait le droit des hommes"14. Le sentiment de propriété est flagrant : le suffrage est un droit qui pourrait éventuellement se partager, mais sur le mode séparatiste, identitaire, chacun gardant la spécificité d'un vote qui ne saurait se définir

\footnotetext{
${ }^{10}$ Jean Philip, op. cit.

${ }^{11}$ François Albert, intervention dans la séance du 21 novembre 1922 ; sénateur de la Vienne de 1920 à 1927, membre du groupe de la gauche démocratique, ce futur ministre de l'Instruction publique (1924) connu pour son éloquence, s'est fait remarquer dans le débat sur le rétablissement des relations diplomatiques avec le Saint Siège (1921). En 1922, il vote contre la discussion des articles.

${ }^{12}$ Alexandre Bérard, intervention au Sénat dans la séance du 16 novembre 1922.

${ }^{13}$ On peut parler de pensée sexualiste lorsque l'analyse de la spécificité politique d'un groupe est directement reliée à l'appartenance sexuelle de ses membres ; conceptuellement, elle s'apparente à la pensée dite "sexiste", d'un usage certes plus courant en sciences humaines mais également plus délicat à manier en raison du grand nombre de connotations féministes et donc partisanes qui l'accompagnent généralement. Par souci de ne pas grever d'emblée l'analyse du discours sur le suffrage des femmes des postulats qui fondent cette pensée, nous préférons employer ce terme qui n'a pas encore d'histoire. Sur l'émergence historique d'une pensée sexualiste, voir infra, note 30.

${ }^{14}$ Aussi propose-t-il de restreindre fortement le projet en n'accordant le droit de suffrage qu'aux femmes de plus de trente ans. Fernand Merlin, intervention dans la séance du 16 novembre 1922 au Sénat. Fernand Merlin, sénateur de la Loire de 1920 à 1937, est un membre du comité directeur du groupe de la gauche démocratique ; très impliqué dans le travail législatif, c'est aussi un ancien député de 1919 qui a voté pour le suffrage féminin. En 1922, il vote bien sûr pour la discussion des articles.
} 
autrement que par son expression sexuée. Car nul n'invoque la nécessité de préserver le vote comme expression d'une universalité que 1"'hégémonie féminine" ${ }^{15}$, seule à rester déterminée par son sexe, viendrait mettre en danger. Il n'y a pas d'une part la République neutre et universelle et d'autre part les femmes ; mais deux groupes sexués qui s'affrontent (ou s'affronteraient, la mise à l'écart permettant de préserver le statu quo).

\subsection{La conviction d'une opposition féminine (cause)}

Que les femmes votent comme des femmes n'aurait peut-être pas tant gêné les sénateurs de la gauche démocratique s'ils avaient pu compter sur leur soutien électoral : mais les sénateurs, qu'ils soient de droite ou de gauche, pro- ou anti-suffrage, supposent qu'il s'exercera massivement contre la gauche démocratique. En réalité, la plupart en sont même convaincus. C'est que tout, de la littérature à l'actualité politique, en passant par les expériences plus immédiates et personnelles, contribue à renforcer l'idée que les femmes sont restées, malgré leur instruction républicaine, "sur les genoux de l'Eglise". Pour ce qui concerne cette étude, nous insisterons plus particulièrement sur l'expérience électorale des parlementaires, en laissant de côté $l^{\prime}$ 'héritage intellectuel républicain sur la question, bien connu ${ }^{16}$, et le rôle qu'a pu jouer l'implication toute récente de l'Eglise sur le terrain du suffrage féminin, qui mériterait une étude à part entière ${ }^{17}$.

Deux anecdotes permettent de mesurer l'importance de l'expérience personnelle dans le positionnement de certains sénateurs sur la question du vote féminin : celle rapportée dans ses mémoires par Jean Philip, candidat malheureux de la gauche radicale aux élections législatives de 1919 ; et celle vécue à peu près à la même époque par Jules Jeanneney, maire d'une petite commune de l'est, qui s'estima lui aussi victime d'une cabale de "l'électorat" féminin.

L'expérience vécue par Jean Philip ${ }^{18}$ a pour cadre les élections législatives de novembre 1919. Les radicaux, accusés de n'avoir su "ni prévoir, ni empêcher, ni raccourcir la guerre" ${ }^{19}, \mathrm{~s}^{\prime}$ attendent à un désastre électoral. Face à eux, un grand nombre de candidats conservateurs qui ont l'avantage d'avoir "un bras, une jambe, un œil, une main, un doigt de moins que leurs concurrents" ${ }^{20}$ et qui "sans rien connaître des questions politiques" vont effectivement remporter les élections dans la plupart des départements, constituant la fameuse "Chambre bleu horizon". Pour analyser sa défaite, le candidat

\footnotetext{
${ }^{15}$ Ibid.

${ }^{16}$ Cf. René Rémond, L'Anticléricalisme en France de 1815 à nos jours ， Paris, Fayard, 1976 (nouvelle édition revue et augmentée, 1999) ; sur un aspect plus particulier, mais aussi une des figures les plus imposantes de la République, cf. J. Cabanis, Michelet, le prêtre et la femme, Paris, Gallimard, 1978.

${ }^{17}$ A partir de 1919, le camp catholique, que ce soit à travers la "Chambre bleu horizon" (instauration de la fête de Jeanne d'Arc) ou les déclarations pontificales (Benoît XV se déclare favorable au vote des femmes), donne à la gauche toutes les raisons de s'inquiéter en s'impliquant assez soudainement dans l'intégration politique des femmes. Cf. Claude Langlois, "Catholiques et laïcs" in Pierre Nora (dir.), Les lieux de mémoire, III. Les France, 1. Conflits et partages, Paris, Gallimard, 1992 ; ainsi que Francine Muel-Dreyfus, Vichy et l'éternel féminin. Contribution à une sociologie politique de l'ordre des corps, Paris, Ed. du Seuil, 1996.

18 Après avoir perdu les élections législatives, Jean Philip se fera élire en 1920 comme sénateur du Gers ; dans le débat de 1922, il fera un long discours en faveur d'un droit de suffrage restreint pour les femmes, débat que nous aurons l'occasion de citer à plusieurs reprises.

${ }^{19}$ Jean Philip, "Souvenirs d'un parlementaire désabusé", in La France active, no 148 à 171, 1935-1936., nº 154, mars-avril 1936.

${ }^{20}$ Georges Buisson, La Chambre et les députés, Paris, 1924, p. 72.
} 
malheureux met cependant en cause un autre adversaire, dont l'influence aurait joué un rôle important dans le vote exprimé par les électeurs : ce sont les femmes. Selon Jean Philip, qui mène sa campagne dans le midi toulousain, s'il a perdu les élections, cet hiver là, c'est qu'à "certains soirs, sous les vieilles halles, et dans la pénombre de chais ou de granges mal éclairées, elles prenaient parfois des airs farouches de tricoteuses. Sans qu'elles eussent à participer au vote, nous étions sûrs d'avance qu'elles l'influenceraient fâcheusement. Et c'est bien ce qui finalement arriva. (...) Sans être positivement un désastre, le résultat, néanmoins, fut piteux." ${ }^{21}$ Et en effet, les radicaux et les socialistes perdent un nombre considérable de sièges, tandis que les candidats du Bloc national obtiennent pour la première fois la majorité à la Chambre des députés.

Jean Philip est à cette époque l'ancien secrétaire et l'admirateur inconditionnel de Ferdinand Buisson, fondateur du parti radical qui assura un soutien des plus constants à la cause des femmes ${ }^{22}$. De cette collaboration, il a apparemment gardé une certaine fidélité à la doctrine féministe, puisqu'il continue de s'en réclamer, comme sénateur, dans le débat de $1922^{23}$. Mais c'est là que l'on voit toute l'ambiguïté des radicaux sur la question des femmes quand, à l'instar de Jean Philip, ils défendent à la fois la justesse du principe d'un droit de suffrage féminin (voire la certitude de son avènement) et la nécessité d'en freiner la mise en oeuvre. Pris entre l'idée qu'il approuve d'un point de vue philosophique et les conséquences dont il a luimême pu (ou cru) mesurer la force, l'élu de la gauche radicale fait le choix, comme la majorité de ses condisciples au Sénat, de ne pas céder au sirènes de la "métaphysique" pour privilégier le pragmatisme et le sens des responsabilités en ne proposant qu'un suffrage restreint pour les femmes.

A la même époque, se déroule une tentative de suffrage universel dans une petite commune de l'Est de la France ${ }^{24}$ qui se solde par un échec tout aussi retentissant pour son maire, Jules Jeanneney ${ }^{25}$. La différence avec l'expérience rapportée par Jean Philip tient au degré de responsabilité des femmes. Alors que pour le candidat malheureux à la députation, celles-ci pouvaient au pire être soupçonnées d'avoir contribué à sa défaite, dans le cas de Jules Jeanneney, les électrices sont perçues comme les seules responsables de l'échec de sa consultation électorale. C'est Louise Weiss qui rapporte cette anecdote racontée parle Président du Sénat, Jules Jeanneney :

"J'étais maire d'une petite commune dans l'Est et pris l'initiative de faire voter les femmes, voici pourquoi et comment. La Saint-Christophe, fête patronale du village, tombait à une époque incommode pour les foires et les

\footnotetext{
${ }^{21}$ Jean Philip, "Souvenirs d'un parlementaire désabusé”, op. cit.

${ }^{22}$ Il faut lire les pages consacrées à Ferdinand Buisson par Jean Philip qui dresse à travers ce "philanthrope", père de l'école laïque qui est aussi l'un des "chefs incontestés du parti radical", le portrait de la probité incarnée. Cf. Jean Philip, "Souvenirs d'un parlementaire désabusé"..., op. cit., n 149, mai-juin 1935.

${ }^{23}$ Tout le discours de Jean Philip mériterait d'être repris, tant il est révélateur de la contradiction qui caractérise la conscience des sénateurs de gauche qui se veulent ouverts sur la modernité tout en se montrant soucieux des conséquences d'une réforme trop brutale; voir son intervention au Sénat, op. cit.

${ }^{24}$ Il s'agit de Rioz, à côté de Besançon, dans la Haute Saône.

25 Jules Jeanneney, sénateur de la Haute Saône de 1909 à 1944, fut très actif à la tribune en même temps qu'un personnage central de la République pendant tout l'entre-deux-guerres, en tant que vice-président puis président du Sénat ; c'est lui qui bloqua la mise à l'ordre du jour de la question des femmes pendant les années où il présida le Sénat (de 1932 jusqu'à la guerre) ; très attaché au maintien des institutions en l'état, hostile à toute réforme, il est l'homme du conservatisme sénatorial par excellence.
} 
moissons. Il était depuis longtemps question d'en changer la date. Ne voulant contrarier personne, je décidai de procéder à la consultation de toute la population. La tendance se confirma en faveur du déplacement de la fête. Mais le dimanche du vote, le curé en chaire déclara qu'un changement de date offusquerait le saint et il objurgua ses paroissiennes de voter contre. Celles-ci lui obéirent en bloc. Est-il vraiment utile d'apporter les voix d'un tel contingent de sottes au scrutin ?"26

Lors de ce court entretien où la féministe était venue lui demander son soutien, celui qui était alors le second personnage de l'Etat avait reconnu qu'"en principe" il serait "juste" d'accorder les droits politiques aux femmes; mais il n'en avait pas moins conclu à l'adresse de Louise Weiss qu'il n'était pas pour son vote ${ }^{27}$. Et le projet d'extension du suffrage en était resté là jusqu'à la fin de la IIIème République.

A partir de ces deux exemples, on voit que s'il est un postulat sur lequel les parlementaires jamais ne fondent d'espoir, c'est celui de la similitude de vote entre les époux. Sur ce point, il ne faut pas se tromper d'époque : l'heure n'est plus à la perspective familialiste ${ }^{28}$, et pas encore à l'amalgame des membres du couple qui caractérisera l'analyse journalistique du comportement électoral des femmes au lendemain de la seconde guerre mondiale ${ }^{29}$. Ainsi, dans le débat de 1922, ne trouve-t-on qu'une seule allusion à l'influence maritale sur le comportement électoral des femmes ; encore est-elle aussitôt ponctuée d'un retentissant "comptez là-dessus !" lancé par un sénateur, sans équivoque quant à un possible espoir de voir les futures électrices voter comme leur mari ${ }^{30}$.

Une idéologie sexualiste est à l'œuvre, c'est certain, dans le débat qui en 1922 mène au refus sénatorial d'étendre le droit de suffrage aux femmes. Est-ce cette idée qui rebute les sénateurs ? Ceux-ci ont-ils à l'esprit qu'un citoyen est un individu neutre qui n'a pas à importer dans la sphère politique une quelconque appartenance, susceptible d'introduire des distinctions et d'interférer avec l'expression de la volonté générale ? Autrement dit, est-ce pour des raisons philosophico-juridiques, tenant à l'emprise du paradigme individualiste sur des esprits majoritairement formés par les facultés

\footnotetext{
${ }^{26}$ Louise Weiss, Ce que femme veut. Souvenirs de la IIlème République, Paris, Gallimard, 1946, p. 88.

${ }^{27} \mathrm{Ibid}$.

${ }^{28}$ Sur cet aspect encore peu connu de la législation électorale antérieure, je me permets de renvoyer à mes travaux de recherche ; Anne Verjus, Les femmes, épouses et mères de citoyens, ou de la famille comme catégorie politique dans la construction de la citoyenneté (1789-1848), thèse de doctorat d'Etudes politiques, EHESS, 1997 — où l'on trouvera notamment une analyse du passage, autour de 1848, de l'idéologie familialiste à la pensée sexualiste (chap. 6) ; voir aussi Anne Verjus, "Femmes et famille dans l'élaboration des droits électoraux de la Révolution de 1789 à la IIIème République", communication au colloque Les droits de l'homme et le suffrage universel, 1848-1948-1998, organisé par le Centre des Droits de l'Homme de la Faculté de Droit de Grenoble, 28-29 et 30 avril 1998..

${ }^{29}$ Cf. Bruno Denoyelle, "Des corps en élections. Au rebours des universaux de la citoyenneté : les premiers votes des

femmes (1945-1946)", in Genèses, no 31, juin 1998, pp. 76-98. Ça n'est pas le lieu, dans le cadre limité d'un article, d'avancer des explications, voire même des hypothèses, concernant les raisons d'une telle évolution. Elles sont le sujet d'un travail de recherche plus général dont le champ d'investigation, déjà bien entamé, devrait s'étendre sur toute la période dite "contemporaine" (1789-1945).

${ }^{30}$ Intervention de Louis Martin, dans la séance au Sénat du 7 novembre 1922, interrompu par un sénateur de la gauche.

Louis Martin, sénateur du Var de 1909 à 1936, membre du groupe de la gauche démocratique, fait partie des sénateurs les plus impliqués sur la question du suffrage féminin, proposant à chaque session, jusqu'en 1935, l'inscription à l'ordre du jour du projet de loi adopté par la Chambre. En 1922, il vote pour la discussion des articles.
} 
de droit $^{31}$, que les femmes n'obtiennent finalement pas le droit de participer aux élections ? Pour répondre à cette question, il est instructif de comparer le débat de 1922 avec celui de 1919.

\section{2. "Les législateurs font les lois pour ceux qui font les législateurs" ${ }^{12}$}

Si l'idéologie sexualiste était en cause dans l'issue négative du débat au Sénat, si c'était en raison d'une quelconque spécificité des femmes, incompatible avec le statut de citoyen, que les sénateurs leur auraient refusé le droit de vote, de deux choses l'une : soit on aurait entendu ceux-ci proposer une vision alternative, notamment individualiste, pour défendre leur position anti-sexualiste. Or, cela n'est jamais le cas. Soit on aurait vu cette idéologie rencontrer une opposition semblable à la Chambre. Or, ce n'est pas le cas non plus, puisque, sur la base de cette même idéologie sexualiste, la Chambre de 1919 adhère de manière enthousiaste au projet de droit de suffrage des femmes.

\section{1 "Nous ne devons pas, ô mes frères dans le sexe, être si fiers de notre ceuvre. ${ }^{133}$}

La guerre a sans doute joué un rôle important dans la progression de la cause féministe au sein des parlementaires ${ }^{34}$. Les députés en mai 1919, comme les sénateurs en novembre 1922, soulignent l'héroïsme et le dévouement féminins à l'arrière comme au front ${ }^{\prime 35}$. Selon Suzanne Grinberg qui assiste alors aux débats, c'est lorsque René Viviani évoque cette participation des femmes à la guerre et le droit qu'elles $\mathrm{y}$ ont gagné à revendiquer leur part de la gestion des affaires publiques qu'une "émotion grave" s'empare et obtient raison de "ceux qui

\footnotetext{
${ }^{31}$ Cf. Yves-Henri Gaudemet, Les juristes et la vie politique de la IIIème république , Paris, PUF, 1970 ; ainsi que François Grèzes-Rueff, La culture des députés français (1910-1958). Essai de typologie .

${ }^{32}$ René Viviani, Le Journal, 30 avril 1906, cité par Vital Gougeon, Du vote des femmes, 1907, p. 104.

${ }^{33}$ Expression de René Viviani, qu'il pose comme un point d'orgue alors qu'il vient de prononcer une diatribe sur les déficiences de la société lorsqu'elle n'est réformée que par les hommes. Cf. René Viviani, discours à la Chambre des députés dans la séance du 20 mai ; ce passage, tel que les lecteurs du Matin l'ont découvert le lendemain, sera reformulé pour les besoins de la publication officielle d'une manière plus sophistiquée, moins parlante mais dont tout le sens a été maintenu : "Nous ne devons donc pas être si fiers de la direction unilatérale que nous avons imposée aux hommes et aux choses, et permettez-moi de dire qu'avec moins d'arrogance nous devons réclamer le privilège exclusif de la direction que, jusqu'ici, nous avons imprimée". Cité par Suzanne Grinberg, Historique du mouvement suffragiste..., op. cit., pp. 141-142.

${ }^{34}$ De ce point de vue, on ne peut en nier l'impact au seul prétexte de la recrudescence soudaine, à la même époque, de la littérature antiféministe. Cf. Ch. Bard, Un siècle d'antiféminisme..., op. cit.

35 René Viviani, discours dans la séance de la Chambre des députés du 20 mai 1919. Député socialiste de la Creuse, il sera élu sénateur de la Creuse de 1922 à 1925. En 1919, c'est un homme politique d'envergure qui a pour ainsi dire terminé sa carrière. Premier Ministre du travail de la République, puis tour à tour Ministre des Affaires étrangères et président du Conseil pendant la guerre, il a indéniablement l'autorité qui explique une part de son succès à la Chambre. Mais c'est aussi un avocat dont l'éloquence attire un auditoire par ailleurs connu pour son avidité de bons mots et de beaux discours. Les témoignages de l'époque montrent un homme qui brilla par une éloquence sans pareille mais dont l'apport politique et la personnalité sont controversés. Il n'en est pas moins un personnage central du régime et son approche du suffrage des femmes, aussi distante soit-elle de la philosophie individualiste républicaine, ne peut être négligée. Suzanne Grinberg, qui assista au débat, commente ce passage du discours par ces mots : "tant d'émotion grave avait eu raison de ceux qui hésitaient encore". Dans la discussion de 1922, il votera pour la discussion des articles. Mais pour des raisons qui mériteraient qu'on s'y penche un peu, il ne répondra pas à l'attente des féministes, en renonçant à intervenir dans la discussion. Cf. Jean-André Tournerie, Le ministère du travail (origines et premiers développements), Paris, Cujas, 1971 , ainsi que Philippe Serre, Éloge de Viviani. Discours prononcé à l'ouverture de la Conférence des avocats, le 30 novembre 1930, Barreau de Paris Ed., 1930, 22 p. Sur l'éloquence sous la IIIème République, voir Nicolas Rousselier, Le Parlement de l'éloquence, Paris, Presses de la FNSP, 1997.
} 
hésitaient encore" ${ }^{136}$. Trois ans après, c'est bien encore en ces termes que l'on analyse le succès du vote féminin dans cette Chambre : le sénateur Fernand Merlin, partisan du projet, évoque la "reconnaissance" pour le devoir accompli par les femmes pendant la guerre ${ }^{37}$.

De toute évidence, ce qu'on appelle alors la "cause des femmes" a progressé depuis le début du siècle ; on trouve certes toutes sortes d'ouvrages autour de 1919 pour afficher leur hostilité à la question du suffrage, mais leur argumentation repose alors plus sur des raisons contingentes telles que les conséquences d'une attribution du vote hic et nunc, que sur le refus philosophique d'instaurer une égalité de droits entre des êtres que la nature à faits inégaux, comme c'était encore le cas dans les années $1890^{38}$. En 1909, soit trois ans après la création à la Chambre d'un Groupe de défense des droits des femmes qui avait recueilli l'adhésion de 200 députés et réalisé en moins de 10 ans la totalité de son programme (certes, modéré) $)^{39}$, Ferdinand Buisson pouvaot constater que l'idée d'un vote des femmes était "sortie de la période des débats théoriques" et qu'il n'était plus permis d'en parler "comme d'un rêve ou d'une élucubration d'utopiste"

En mai 1919, le contexte est donc favorable à la prise en considération de la question du vote des femmes; à cet égard, le fait qu'elle soit débattue à la Chambre peut être considéré comme un des signes de cette banalisation du principe, et de sa popularité. Mais si le principe $\mathrm{du}$ "droit au droit" est acquis parmi la plupart des parlementaires républicains, sa mise en application est loin d'aller de soi ; il y a là une véritable lutte à mener, qui se traduit par ce débat si passionné de mai 1919.

Louis Andrieux, qui est le premier à proposer d'aller au-delà du projet trop timoré de la Commission en accordant aux femmes un droit de vote intégral, justifie son texte en arguant du besoin qu'a cet électorat de défendre ses intérêts dans sa "lutte pour la vie" :

\footnotetext{
${ }^{36}$ Suzanne Grinberg, Historique du mouvement suffragiste..., op. cit., p. 142.

${ }^{37}$ Fernand Merlin, intervention devant le Sénat, dans la séance du 16 novembre 1922 ; à la même époque, un autre témoin, Joseph Barthélémy, juriste et bientôt député qui aura son rôle dans l'évolution de la question débute un cours sur le vote des femmes en constatant que, loin de renforcer l'inégalité entre les sexes, la guerre a "fait accomplir à la cause de l'égalité des sexes, dans un grand nombre de pays, de rapides et extraordinaires progrès". Joseph Barthélémy est une figure étonnante, justement typique, du parlementaire de la IIIème République ; sur la question des femmes, son cours (ainsi que son rapport de 1923) cristallise toute l'ambiguïté du discours juridique en reconnaissant d'une part le comportement conservateur des femmes, et en souhaitant que cet argument n'entre pas en ligne de compte lorsque viendra le moment de discuter la question ; Ferdinand Buisson, dans son rapport de 1910, avait fait le même constat. Nous verrons plus loin comment les parlementaires ne peuvent répondre à ces injonctions qualifiées de "métaphysiques" autrement qu'en mettant l'accent sur leur propre sens du concret, leur pragmatisme et leur sens des réalités. Voir Joseph Barthélémy, Le vote des femmes, 1917-1918, ouvrage couronné par l'Académie des Sciences morales et politiques (prix du Budget), Cours professé à l'Ecole des Hautes Etudes sociales pendant l'année 1917-1918, Paris, Alcan, 1920,p. V.

${ }^{38}$ Un article d'Edmond Villey, professeur d'économie politique, doyen de la faculté de droit de Caen, fondateur de la Revue d'économie politique, reflète assez bien les opinions les plus couramment admises sur la question du suffrage des femmes à cette date. Son argumentaire est une affirmation autoritaire, basée sur le postulat qu'hommes et femmes sont différents en droits comme en nature. Voir Edmond Villey, "Les droits de la femme", in Revue du droit public et de la science politique en France et à l'étranger, juillet 1896, notamment p. 42.

${ }^{39}$ Selon Véronique Antomarchi, le Groupe créé en 1906 par Henry Chéron sur la base d'un programme de défense de la famille et de lutte contre la dénatalité devra son succès (200 députés y adhèrent et contribuent à la réalisation du programme avant la première guerre mondiale) à la modération de son initiateur, Henry Chéron. Cf. Véronique Antomarchi, La politique familiale en France sous la Troisième République (1870-1914), thèse d'histoire de l'Université de Paris I-Panthéon-Sorbonne, 1995, pp. 134-135 (thèse publiée aux éditions de L'Harmattan en 2000 sous une forme abrégée).

${ }^{40}$ Ferdinand Buisson, Le vote des femmes, Paris, 1911. Son rapport fut déposé à la Chambre le 16 juillet 1910 ; Suzanne Grinberg le considère comme "le premier ouvrage documentaire sur la question du suffrage féminin dans la plupart des pays du monde au point de vue doctrinal et politique.” Suzanne Grinberg, Historique du mouvement suffragiste..., op. cit., p. 110.
} 
"Pour cette infériorité de la femme, pour cette injustice dans la répartition de la rémunération du travail, il y a une autre raison, c'est que l'homme, lui, pour la défense de ses intérêts et de ses droits, il est citoyen, il a le bulletin de vote, il a ce fragile papier, une puissance qui met dans sa main les élus, leurs intérêts, leur réélection. Dans une démocratie ceux qui ne votent pas ne comptent pas; et c'est une des raisons profondément humaines, pour lesquelles je vous demande de donner à la femme le moyen de défendre son existence, celle de ses enfants, l'avenir des siens. ${ }^{\prime 41}$

Le contre-projet d'Andrieux emporte le vote de la quasi-unanimité des députés ; mais c'est plus probablement l'éloquence de René Viviani qui décide ceux-ci à aller contre l'avis des deux commissions qui avaient recommandé d'en rester à un suffrage restreint ${ }^{42}$. Le contexte de la guerre y sert encore d'argument, cette fois pour souligner, en creux, la responsabilité des hommes dans les tueries dont le souvenir poursuit certains jusque dans leur chair ${ }^{43}$. Viviani y inscrit son discours pour donner à l'argument sexualiste, c'est-à-dire à la défense du droit des femmes sur la base de leur spécificité, toute sa puissance évocatrice :

"...l'intervention des femmes dans la vie publique à l'heure actuelle aurait pour conséquence de troubler la paix publique que, vous le savez par expérience, les hommes ont toujours aidé à fonder et à fortifier. ${ }^{\text {"14 }}$

L'ironie de l'allusion n'échappe certainement à personne ${ }^{45}$.

La guerre n'est évidemment pas le seul terrain de lutte entre les sexes ; pour l'ancien avocat, il y a aussi, et peut-être en premier lieu, celui du mariage et de la famille. A cet égard, le seul moyen pour que les femmes cessent de venir "pleurer dans [les] cabinets d'avocats" serait de leur donner les moyens de défendre leurs propres intérêts d'épouses et de mères dans la lutte qui, depuis longtemps, les oppose aux hommes :

"Dans nos cabinets d'avocat, nous avons reçu bien des confidences mouillées de larmes. Des femmes tombées au fond de la détresse morale nous demandaient des armes, et quand nous leur disions que le code était vide, elles s'étonnaient, elles s'indignaient, et nous avions envie de leur répondre qu'elles ne commençaient à se soucier du malheur des autres que quand le bonheur leur échappait. ${ }^{46 "}$

Pour celui qui bien des années auparavant déclarait déjà que "les législateurs font les lois pour ceux qui font les législateurs" ${ }^{47}$, les

\footnotetext{
${ }^{41}$ Intervention d'Andrieux dans la séance du 20 mai 1919 à la Chambre des députés.

${ }^{42}$ L'intervention de Viviani est manifestement attendue et écoutée avec attention.

${ }^{43}$ René Viviani avait été très affecté, dit-on, par la mort au champ d'honneur de son beau-fils. Combien d'autres avaient perdu des enfants dans la bataille ? Les biographies des députés et sénateurs de la période laissent entrevoir ces drames qui ont touché tant de familles et leur effet dévastateur sur ces pères.

${ }^{44}$ René Viviani, intervention à la Chambre dans la séance du 20 mai 1919.

${ }^{45}$ Pour ceux qui parmi les députés ne l'auraient pas saisie, Viviani se fait plus explicite en opposant à plusieurs reprises le vide ou les désastres qui accompagnent l'œuvre accomplie par les hommes et les perspectives de pacification et de renouveau auxquelles l'inclusion des femmes donnerait un espoir de réalisation.

${ }^{46}$ René Viviani, intervention citée.

${ }^{47}$ En 1900, Viviani défendait déjà cette position : "toutes les lois que nous pourrons proposer seront vaines si pour accroître et défendre ces lois, les femmes ne sont pas armées du bulletin de vote. Jusque-là, elles n'obtiendront de la générosité des hommes, de leur esprit de justice ou de leur amour du paradoxe, que des réformes partielles, quelques menues modifications
} 
femmes ne trouveront jamais de représentants de leur cause chez les hommes tant qu'elles ne contribueront pas elles-mêmes aux élections. On est loin du concept de "représentant de la Nation" : les femmes, si elles sont toujours vues comme des épouses, ne le sont plus du point de vue traditionnel où la hiérarchie englobait ses parties en un tout unifié $^{48}$, mais d'un point de vue classiste/sexualiste où les parties en lutte voient leurs intérêts s'opposer; en outre, politiquement, la femme n'est plus une "épouse en puissance" ; elle est d'abord une personne sexuée dont une des situations juridiques possible, celle d'épouse, peut l'obliger à se défendre contre l'homme, d'abord représentant de son propre sexe et accessoirement son mari.

Au-delà de ses propres intérêts, selon Viviani, la femme comme électrice est destinée à apporter ses compétences naturelles en matière de protection de la famille, d'hygiène et de lutte contre la dénatalité. On retrouve là des thèmes caractéristiques des campagnes anglosaxonnes en faveur du suffrage féminin qui, à la même époque, s'appuient largement sur le postulat de "la différence des femmes par rapport aux hommes en tant que sexe (gender) et non [sur] leur équivalence en tant qu'individus." ${ }^{49}$

La décision de la Chambre se fait sur des discours ne reposant pas sur les arguments que l'on pourrait attendre d'une assemblée composée majoritairement de juristes et de députés radicaux et radicaux socialistes $^{50}$, a priori plutôt attentifs à faire respecter les droits de l'individu, donc peu enclins à reconnaître des intérêts divergents dans la société. Or, l'argumentaire utilisé par Louis Andrieux ou René Viviani repose non pas sur le droit des femmes au droit de vote, mais sur leurs intérêts en tant que catégorie particulière dans la nation. Ils se distinguent habilement (car sciemment) du discours juridique ambiant qui lui, pose le principe d'un droit au droit de suffrage, reposant sur l'appartenance des femmes à la société ${ }^{51}$.

\subsection{Un vote cynique?}

Il existe une thèse selon laquelle les députés auraient émis un vote "cynique", c'est-à-dire délibérément voté pour le projet le plus radical dans le but de nuire plus sûrement à la cause des femmes. Cette thèse, d'abord répandue pour les besoins du groupe de la Gauche démocratique, a été reprise par les historiens pour expliquer ce vote si surprenant en faveur du suffrage féminin. En réalité, elle ne repose sur rien, sinon sur cette rumeur qui profite surtout aux opposants du projet, et des calculs qu'une simple vérification n'avère pas.

La rumeur selon laquelle la Chambre des députés, en 1919, c'est-à-dire "à la veille de sa séparation, expirante, presque morte, avait voulu

au Code civil ou au Code du commerce... car les législateurs font les lois pour ceux qui font les législateurs." Le Journal, 30 avril 1906, cité par Vital Gougeon qui dans sa thèse datée de 1907 ajoute : "en parlant ainsi, M. Viviani n'a fait qu'exprimer et résumer l'opinion commune." Cf. Vital Gougeon, Du vote des femmes, 1907, p. 104.

${ }^{48} \mathrm{Cf}$. Anne Verjus, Les femmes, épouses et mères de citoyens..., op. cit.

49 Pierre Rosanvallon, Le sacre du citoyen..., op. cit., p. 405.

50 Les socialistes représentent $22 \%$ de la Chambre de 1919 ; les radicaux et $\quad$ radicaux socialistes, $36 \%$; les républicains conservateurs, $29 \%$ et la droite, $11,5 \%$. Pourcentages établis à partir des données de S. Hause \& A. Kenney, Women's Suffrage..., op. cit.

${ }_{51}$ Cf. un ouvrage représentatif, celui de Charles Turgeon, Le féminisme français, Paris, 1902, 500 p. 
nous tendre un piège ${ }^{52}$ et se ménager, à nos dépens, je ne sais quelle popularité sans gloire, en conservant aux yeux des femmes le mérite de son vote, mais en nous livrant, sur des instructions plus ou moins vagues et mal définies, une sorte de fantaisie mort-née qu'elle ne se serait pas souciée autrement de voir lui survivre", est selon Antonin Gourju $^{53}$, sénateur partisan du suffrage des femmes, l'un des

"phénomènes d'interprétation les plus extraordinaires" qu'il ait jamais vus. Et en effet, la lecture des débats de 1922 permet de vérifier la prégnance de cette idée que l'on a répandue "dans la presse, dans les couloirs et même ailleurs" ${ }^{154}$ selon laquelle le vote de la Chambre n'a été qu'un "vote de façade" ${ }^{n 5}$. La plupart des intervenants hostiles au projet, partisans modérés comme Jean Philip et André Porteu ${ }^{56}$, ou opposants comme Alexandre Bérard et Jacques Régismanset, font allusion au climat particulier dans lequel a été voté le contre-projet d'Andrieux : les uns parlent de vote "un peu emballé" 57 , de "surprise" ${ }^{58}$, reconnaissant tout de même que c'était compréhensible au "lendemain de l'agitation tragique de la guerre" mais insinuant aussi qu'avec trois années de recul, il conviendrait de revenir sur le projet dans des dispositions plus modérées ; un autre parle des députés comme "d'idéalistes", de "révolutionnaires qui se croyaient, une fois de plus, à la veille du grand soir", qui "ne se faisaient guère d'illusion sur le sort final du projet" et parmi lesquels se trouvaient un très grand nombre de "naufrageurs" ${ }^{\text {"59 }}$. Cette thèse, selon laquelle la plupart des députés n'ont exprimé qu'un vote cynique, est très répandue dans les rangs de la gauche démocratique ; elle l'est d'autant mieux, peut-être, qu'elle est un moyen d'invalider ce vote émis par une très forte majorité de représentants de la nation d'une part, et de gauche d'autre part.

Cette rumeur, qui sert si bien les intérêts des sénateurs de la Gauche démocratique, a par la suite été reprise par l'historiographie ; or, quoiqu'elle ait cet avantage d'offrir une explication convaincante à la contradiction entre les votes des députés et des sénateurs, elle ne résiste pas à la vérification des éléments chiffrés sur lesquels elle repose $^{60}$.

Steven Hause et Anne Kenney ont cherché à valider cette thèse dans un ouvrage dont les conclusions sont depuis régulièrement reprises ${ }^{61}$. Selon eux, la thèse de la "cynique machination politique" est principalement étayée par deux éléments : premièrement, par le simple constat qu'une majorité de radicaux ont voté pour le suffrage féminin à la Chambre des députés alors qu'ils sont une majorité à voter contre au

\footnotetext{
${ }^{52}$ Il s'agit des sénateurs.

${ }^{53}$ Antonin Gourju, sénateur du Rhône, est inscrit au groupe de la Gauche républicaine. En 1922, il vote pour la discussion des articles.

${ }^{54}$ Antonin Gourju, intervention dans la séance du 14 novembre 1922, au Sénat.

55 Ibid.

${ }^{56}$ André Porteu, dit Porteu de la Morandière, sénateur d'Ille et Vilaine de 1920 à 1932, est inscrit au groupe de la gauche républicaine ; en 1919, député, il avait voté contre le suffrage des femmes ; en 1922, il vote pour la discussion des articles.

57 Jacques Régismanset, intervention dans la séance du 21 novembre 1922, au Sénat.

${ }^{58}$ Alexandre Bérard, intervention dans la séance du 16 novembre 1922, au Sénat.

${ }^{59}$ Porteu de la Morandière, intervention dans la séance du 21 novembre 1922, au Sénat.

60 A partir de la liste des sénateurs de 1922, ont été systématiquement répertoriés, pour les besoins de cette vérification, leur situation électorale en 1919, leur vote sur la question du suffrage des femmes s'ils étaient députés à cette date, leur appartenance politique, leur vote en 1922, en se fondant sur les listes fournies par les Archives parlementaires et les éléments biographiques du dictionnaire de Jean Jolly, Dictionnaire des parlementaires français..., op. cit.

${ }^{61}$ Steven Hause et Anne Kenney, Women's Suffrage..., op. cit., p. 143.
} 
Sénat ; deuxièmement, par une analyse du vote des sénateurs de 1922 qui en 1919 étaient encore députés.

Le premier élément repose sur un postulat contestable, à savoir qu'une appartenance politique commune devrait suffire à déterminer les votes des individus. Sur ce postulat, on établit que même en l'absence de convergence visible comme c'est manifestement le cas entre les radicaux de 1919 et ceux de 1922, il doit quand même y en avoir une, mais cachée. D'où la thèse du cynisme, qui réintroduit de la cohérence là où elle n'apparaît pas au premier abord.

Le deuxième élément, censé confirmer cette thèse, réside dans l'analyse comparée des votes de ceux qui ont participé aux deux votes. Selon Hause et Kenney, une majorité des sénateurs de 1922 étaient députés en 1919 ; parmi les 12 qui ont voté pour le suffrage féminin lorsqu'ils étaient députés, on ne trouverait qu'une minorité (5 exactement) à avoir maintenu leur suffrage en 1922. Ils seraient donc une majorité (7/12) à prouver, par leur refus comme sénateurs d'entériner leur décision de députés, qu'ils n'avaient choisi le contreprojet que pour mieux fragiliser la réforme du suffrage en misant sur le conservatisme et le positionnement bien connu du Sénat sur cette question.

De fait, on ne trouve pas une majorité de sénateurs qui seraient d'anciens députés de la Chambre de 1919, mais moins de $20 \%$ de l'ensemble des sénateurs ${ }^{62}$, soit 58 . Or, parmi ces 58 "sénateurs anciens députés", on n'en trouve pas 12 à avoir voté pour le contreprojet en 1919 , mais $26^{63}$ (soit $45 \%$ ) ; et parmi ces 26 , ils ne sont pas 5, mais 18 à avoir voté pour la poursuite de la discussion en 1922. Autrement dit, 69\% des députés présents lors du vote de 1919 et devenus sénateurs en 1922 ont maintenu leur vote en faveur du suffrage des femmes. Si le cynisme concerne malgré tout une partie des députés, ce n'est pas dans ce groupe des futurs sénateurs qu'on le trouve.

Si maintenant on examine le comportement de ceux qui, dans cette catégorie de sénateurs-anciens-députés, appartiennent au groupe de la gauche démocratique, on s'aperçoit que sur 40 d'entre eux, 28 ont confirmé leur vote de 1919, qu'il soit positif ou négatif ; dans l'un et l'autre cas, on ne peut invoquer le cynisme, celui-ci ne pouvant par définition concerner que ceux qui, en 1919 ont voté pour et en 1922 auraient révélé leurs intentions réelles de "naufrageurs" du projet en votant contre. Or, ils sont 6 à avoir ainsi voté, sur les 13 futurs sénateurs de la gauche démocratique à avoir voté pour en 1919. Parmi les 12 anciens députés conservateurs, on n'en trouve qu'un à avoir exprimé un vote cynique. En comptant encore deux anciens députés dont l'appartenance politique est plus incertaine, on parvient à un total général de 58 anciens députés, dont les trois quarts ont maintenu leur vote de 1919 en 1922, et seulement 7 (12\%) ont manifesté ce qu'on peut appeler une attitude cynique. Si l'on en juge par ce comportement

\footnotetext{
${ }^{62}$ Parmi les sénateurs de 1922 qui étaient députés en 1919, 40 sont de la "gauche démocratique", 4 sont socialistes, 12 sont conservateurs et 2 sont d'appartenance indéterminée, ce qui fait un total de 58 sur 291 sénateurs qui se sont exprimés (soit $19 \%$ exactement).

${ }^{63}$ La liste est aisée à faire, puisqu'elle est fournie par Antoine Gourju dans son discours de 1922. Voir son intervention au Sénat dans la séance du 14 novembre 1922. Au sein de cette liste, Gourju cite deux sénateurs, Etienne et Andrieu, que je n'ai pas pris en compte. Le premier parce qu'il est décédé avant le vote de novembre ; le second parce qu'il ne prend pas part au vote (en 1919, député, il avait voté contre le suffrage féminin).
} 
des anciens députés devenus sénateurs, on doit constater que la thèse du vote cynique ne tient pas ${ }^{64}$.

LE VOTE DES SÉNATEURS ANCIENS DÉPUTÉS DE 1919

Progressistes Conservateurs Indéterminés Total

\begin{tabular}{lccccc}
\hline $\begin{array}{l}\text { Partisans } \\
\text { inconditionnels } \\
\text { (pour en 1919 et }\end{array}$ & 4 & 8 & 6 & 2 & $\mathbf{2 0}$ \\
en 1922) & & & & & \\
\hline
\end{tabular}

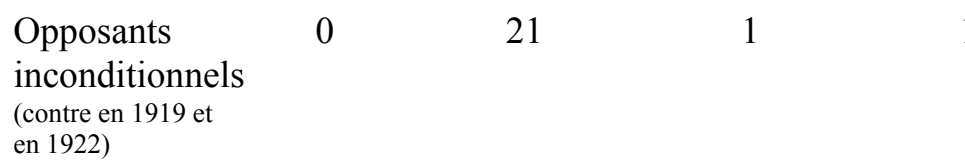

123

en 1922)

\begin{tabular}{llllll}
$\begin{array}{l}\text { Hésitants } \\
\text { (contre en 1919 et } \\
\text { pour en 1922) }\end{array}$ & 0 & 5 & 3 & 0 & $\mathbf{8}$ \\
\hline $\begin{array}{l}\text { Cyniques } \\
\text { (pour en 1919 et } \\
\text { contre en 1922) }\end{array}$ & 0 & 6 & 1 & 0 & $\mathbf{7}$ \\
\hline TOTAL & 4 & 40 & 11 & 3 & $\mathbf{5 8}$
\end{tabular}

Pour tous les parlementaires, et quelle que soit leur position sur la question du suffrage, les femmes constituent un électorat dont le comportement homogène fait une véritable masse à intégrer au sein du suffrage masculin. Les femmes vont voter comme des femmes, penset-on à droite comme à gauche : certains le craignent, d'autres l'appellent de leurs vœux. Mais si, à cette date, on emploie parfois le terme d'individu pour justifier leur droit au droit, c'est d'un "individu féminin" qu'il s'agit. Au-delà de l'abstraction du droit au droit de tout être humain, on sent bien que cet individu-là ne votera pas "comme un homme" ; il ne se contentera pas de doubler l'électorat, mais apportera une masse au comportement électoral spécifique, sexué et globalement conservateur. L'argumentaire du droit au droit ne suffit pas à convaincre une Assemblée de l'opportunité d'une réforme qui attend depuis si longtemps ; pour la faire passer, il faut arguer du besoin des femmes, dans la société d'aujourd'hui, d'une représentation sans laquelle elles sont dominées, sans moyen de défense. Le besoin, contrairement au droit, ne s'argumente pas. Surtout, il s'impose comme un fait que les députés n'ont qu'à entériner d'une part, et combler par la loi d'autre part. De métaphysique, sans utilité sociale immédiatement visible, la loi devient concrète, nécessaire et passe

\footnotetext{
${ }^{64}$ En outre, en montrant combien l'appartenance régionale influence le vote des députés, pour appuyer la thèse qu'ils n'ont voté que selon leur sentiment/mentalité, les historiens invalident celle qu'ils défendent par ailleurs. En effet, si le vote de la Chambre en 1919 relève vraiment d'une machination politique, il est inutile de rechercher les critères du vote pro-féminin, le propre de la machination étant de relever de la tactique et non de déterminations socio-politiques telle que l'appartenance à la région du Nord ou aux régions plus latines et catholiques.
} 
dans l'ordre du politique, c'est-à-dire du changement possible, visible, efficace, nécessaire enfin.

Ça n'est pas le postulat de la différence de sexe comme structuration de l'espace politique qui est en cause dans l'échec de 1922 puisque les parlementaires le partagent qu'ils soient pour ou contre et que les députés adoptent le projet d'un suffrage intégral à la suite de discours très ouvertement sexualistes, comme ceux de Louis Andrieux et René Viviani. Omniprésente, traversant tout l'échiquier politique, la conviction sexualiste est une idéologie dominante dont les principes de base, sorte de sens commun sur lequel les pro et anti-suffrage s'entendent, ne permettaient pas de prévoir en soi l'issue des débats. Sauf à connaître le contexte politique de l'époque, l'état des forces en présence, et l'hostilité de la gauche radicale à l'intrusion massive dans le corps électoral d'un élément potentiellement destructeur de son propre pouvoir. Mais on quitte alors le domaine de la philosophie de la République pour entrer dans celui de l'opportunisme et des rapports de force, qui ont joué un rôle déterminant dans l'échec du projet au Sénat.

\section{Une politique du pragmatisme}

Ce qui détermine les conclusions opposées des deux chambres, en dépit de cette idéologie commune, est en liaison avec les situations respectives des parlementaires en tant que députés ou sénateurs. Ce sont elles qui, plus que les opinions ou les positions philosophiques (toujours difficiles à évaluer dans le cas d'un vote sans justification) incitent les uns et les autres à voter pour ou contre le droit de suffrage des femmes. Leur poids sur le choix des parlementaires, exception faite de quelques personnalités qui se font un devoir de défendre le principe, confirme que la résistance au féminisme, dans l'enceinte du Sénat en 1922, relève moins d'une attitude misogyne ou d'une philosophie de l'individu, que d'un moyen de conservation du pouvoir.

Pour justifier leur opposition, les sénateurs ne tarissent pas d'éloges sur eux-mêmes, tentant ainsi de conférer à leur position si ostensiblement opportuniste, un peu de la dignité de leur fonction. Audelà du pragmatisme et de la responsabilité qui sont sans doute les deux qualificatifs les plus fréquemment cités, ce sont le courage et le devoir de maintenir l'ordre dans la République qui définissent le mieux, à leurs yeux, le rôle qui dans la République leur est imparti. Jean Philip, dont on ne finit plus de souligner l'attitude ambiguë dans ce débat et qui se fait une si haute idée de la fonction sénatoriale ${ }^{65}$, admire ceux qui "avec une sincérité à laquelle [il rend] hommage, à la sommation des femmes parties à la conquête de leur droit, ont le courage de répondre "non", à la condition que ce non soit dit sans brutalité" $^{\prime 66}$. Pour un partisan du projet, reconnaître le courage et la sincérité de ses adversaires n'est pas de la meilleure stratégie. Mais on sait que Jean Philip balance entre les deux positions, lui qui propose un suffrage restreint ; aussi, contre la cause du suffrage féminin qu'il croit défendre, donne-t-il en réalité à tous ceux qui ont encore un

\footnotetext{
${ }^{65}$ Cf. Jean Philip, op. cit., n ${ }^{\circ}$ 160, mars-avril 1937, sur le rôle du sénateur comme modérateur et gardien de la République.

${ }^{66}$ Jean Philip, intervention au Sénat, op. cit.
} 
doute, toutes les raisons de s'abstenir de contrer le vœu de la commission. Aux féministes qui disent : "Messieurs les sénateurs, jetez-nous dans la mer de nos droits politiques. Ici le plongeon seul peut apprendre à nager", le sénateur répond :

\begin{abstract}
"Oui, mais il y a des plongeons dont on ne revient jamais, et il $y$ en a d'autres qui font chavirer la barque. Or, la barque, c'est le régime républicain, et nous en tenons les rames; et, tant que les rames n'auront pas été arrachées des mains débiles des vieux messieurs que nous sommes (...) - car vous savez que c'est sous ce vocable peu flatteur que nous sommes jugés et appréciés dans certains milieux féministes (Rires) - nous avons le droit et même le devoir de surveiller les apprenties nageuses et de nous opposer à de dangereuses acrobaties. Après tout, qui donc nous ferait un reproche, républicains, de vouloir garder intacte la République ? ${ }^{167}$
\end{abstract}

Ce devoir de maintenir l'ordre revient à plusieurs reprises; face à cet autre devoir qui serait "d'assurer le maximum d'extension au principe du suffrage universel" ${ }^{\prime 68}$, il semble qu'il pèse d'un poids plus lourd aux yeux des sénateurs qui se pensent investis de la sagesse politique de la nation, y compris au risque de mécontenter une opinion à laquelle ils font, de toute façon, peu référence.

En 1919, les députés avaient semblé osciller entre la curiosité pour les uns et l'affirmation plus philosophique d'un principe pour les autres ${ }^{69}$. C'est du moins le point de vue des sénateurs, tel Jean Philip, encore, qui suggère de donner le droit de vote aux femmes "pour les seules assemblées législatives, afin que nos jeunes et courageux collègues de la Chambre des députés, qui ont voté cette réforme à l'unanimité, puissent en faire sur eux-mêmes la première application, comme ces médecins intrépides, ayant inventé un nouveau sérum, veulent d'abord se l'inoculer. (Rires et applaudissements.)" ${ }^{170}$ L'image qu'il utilise est assez révélatrice de l'atmosphère expérimentale qui a dû entourer le projet d'extension du suffrage aux femmes ${ }^{71}$.

Le "très savant docteur Labrousse", au discours si emblématique des positions de la gauche démocratique ${ }^{72}$, ne manque pas de nous donner à voir sa perception de la dichotomie entre les députés, tous idéalistes, et les sénateurs, pragmatiques par fonction et nécessité. Les premiers sont, selon lui, des "rêveurs" et des "ultra-logiciens" qui défendent une "justice intégrale, totale, à rendre aux femmes, thème facile de déclamations toujours applaudies" ; tandis que les seconds, opposés à "l'immixtion des femmes dans la politique", totalisent "la somme de sens pratique, d'intelligence des instincts, de prudence politique" qui fait préférer la justice qui se "combine avec l'utilité sociale" ${ }^{73}$. Et en

\footnotetext{
${ }^{67} \mathrm{Ibid}$.

${ }^{68}$ François Albert, intervention dans la séance du 21 novembre 1922, au Sénat.

${ }^{69}$ Cf. Porteu de la Morandière, intervention dans la séance du 21 novembre 1922 au Sénat.

${ }^{70}$ Intervention dans la séance du 16 novembre 1922 au Sénat.

${ }^{71}$ Viviani, quant à lui, avait encouragé son auditoire à braver ce qu'il semblait considérer comme une position au moins autant sénatoriale que partisane sur la question : "Nous cherchons avec raison l'harmonie entre les deux assemblées. Cependant la Chambre du suffrage universel a des droits qu'elle ne laissera pas peut-être toujours mettre dans l'ombre, et nous pouvons demander pour nous-mêmes le droit de légiférer en toute indépendance, sans céder au moins par avance." De vifs et quasi unanimes applaudissements accueillent cette déclaration, puis de nouveau c'est le silence attentif". Texte du discours reproduit par Le Matin du 21 novembre 1922.

${ }^{72}$ François Labrousse, sénateur de la Corrèze de 1921 à 1945, est inscrit au groupe de la Gauche démocratique. Son discours a un succès tel que l'orateur y acquiert une petite notoriété parmi ses collègues de la gauche qui n'auront de cesse de le citer avantageusement. Le dictionnaire biographique de Joly indique même que sa réputation d'orateur naquit avec ce discours.

${ }^{73}$ François Labrousse, intervention au Sénat dans la séance du 7 novembre 1922.
} 
effet, tout au long du débat, c'est à qui affichera la plus grande distance à l'égard de la "philosophie", ramenée au rang des "questions d'ordre spéculatif ${ }^{174}$, pour se faire investir de la dignité "d'homme d'Etat" et reconnaître une forme de légitimité à aller aussi ouvertement à l'encontre du projet de la Chambre des députés. Même les défenseurs $\mathrm{du}$ projet qualifient de "responsable" l'attitude hostile de la commission. Ainsi, Louis Martin qui reconnaît que le rapporteur de la commission a pris position contre le suffrage des femmes "avec le sentiment profond de ses responsabilités envers son parti et envers son pays, ${ }^{, 75}$.

Cette opposition entre réalisme et théorie est une préoccupation constante des sénateurs puisque même les partisans du projet refusent leur inscription dans l'ordre des idées pour revendiquer leur part de "pragmatisme". Ainsi Fernand Merlin qui, en se défendant de vouloir faire de la "littérature à cette tribune", reconnaît que "les destinées du pays ne sont point liées à l'idéalisme [mais qu']elles sont surtout commandées par les événements"16 ; ou encore François-Saint-Maur, autre partisan du suffrage féminin, sur le versant conservateur d'un vote familial :

"Seulement, on me dira, et j'accepte l'objection : "Nous ne
sommes pas des théoriciens, nous sommes des réalistes. Un
principe, si bon soit-il, peut avoir et doit avoir souvent des
limites d'application". Soit, mais faut-il encore qu'il y ait à
cela des raisons graves.7",

Cette fonction de responsabilité leur a été historiquement attribuée, puisqu'elle fait partie de la répartition constitutionnelle des charges entre la Chambre basse et la Chambre Haute ${ }^{78}$; c'est également un rôle que la majorité des sénateurs de 1922 endossent avec un sérieux qu'on peut trouver particulièrement en résonance avec leurs intérêts électoraux. Mais il faut dire que les sénateurs de la Gauche démocratique ont de sérieuses raisons d'invoquer leurs fonctions de conservation, face à un électorat qui constitue un réel danger selon eux.

\section{Le sexualisme comme idéologie, l'antiféminisme comme justification et le pragmatisme comme cause (conclusion)}

Que les femmes soient sous l'influence du clergé est en effet une des idées fixes de l'époque. Depuis Michelet, toute une série d'intellectuels ont émis leur jugement sur la question, contribuant à donner à

\footnotetext{
${ }^{74}$ Fernand Merlin, intervention au Sénat, op. cit.

${ }^{75}$ Louis Martin, intervention au Sénat, op. cit. On devine aisément que l'orateur tente par ce biais d'inscrire lui aussi la position féministe hors du champ métaphysique dans laquelle ses adversaires tentent de le cantonner ; mais il prend le risque, ce faisant, de n'avoir plus rien à répondre à l'argument le plus pragmatique qui soit, celui des conséquences électorales de la décision politique. Position d'autant plus malhabile, que Louis Martin n'invoque guère autre chose que la "morale" et la "justice" pour justifier le droit des femmes à une représentation politique ; et d'autant moins convaincante, qu'il donne aux "pragmatiques" toutes les raisons de se féliciter de leur décision, en reconnaissant que lui même, s’il était dans le doute, "si philosophiquement [qu'il soit] engagé à défendre le droit des femmes, [sa] foi peut-être chancellerait.” (Ibid.)

${ }_{77}^{76}$ Fernand Merlin, intervention au Sénat, op. cit.

${ }^{77}$ Sénateur de la Seine inférieure de 1920 à 1940, président de la fédération nationale catholique, François Saint-Maur siège à droite. un sénateur conservateur, catholique, il va de soi que la religiosité des femmes, qu'il avoue, n'est pas une raison assez grave pour repousser la réforme ; aussi vote-t-il pour la discussion des articles. Voir son intervention dans la séance du 16 novembre 1922.

${ }^{78}$ Cf. Jean-Pierre Marichy, La deuxième Chambre dans la vie politique française depuis 1875 , Paris, Librairie générale de droit et de jurisprudence, 1969, $787 \mathrm{p}$.
} 
l'expérience de chacun en la matière une aura de vérité sociologique. Nul n'a, à l'époque, besoin de brandir de statistiques pour affirmer que "la religion n'est plus maintenue dans le monde que par la femme" Quant aux historiens, ils reconnaissent majoritairement aujourd'hui que ce "grand argument que les républicains, radicaux et modérés mettent en avant pour légitimer la mise à l'écart des femmes" ${ }^{80} \mathrm{a}$ eu un impact, mais pour aussitôt le réduire au rang de stratégie ${ }^{81}$. Or, toute stratégie étant au service d'une cause, c'est ailleurs que dans le danger clérical qu'il faudrait chercher celle-ci. Pourtant, nulle part autant que dans ce pays la lutte entre l'Etat et l'Eglise n'est plus forte ; et surtout, nulle part elle n'est autant constitutive et de la République et de l'identité du parti radical qui en a fait sa raison d'être. Bien des travaux ont mis en évidence cette incapacité de la République à affronter l'adversaire autrement que sur le mode identitaire, tragique, permettant de donner à la métaphore, comme celle de la barque qui chavire, la force d'une menace ${ }^{82}$. Le danger d'un renversement de la République pouvait bien être sans fondement; le sentiment de sa force n'en faisait pas moins un acteur du système politique de cette période, qu'il convient de ne pas négliger.

Envisager l'argument anticlérical comme une tactique revient à supposer soit que le but premier des sénateurs était de dénier le droit de suffrage aux femmes en tant que telles (misogynie intrinsèque); soit que, bien plus que la menace de perdre le pouvoir et de devoir faire face à un changement électoral majeur, ce sont des raisons philosophiques qui expliquent leur hostilité (individualisme libéral). La lecture attentive des débats ne permet pas d'adhérer pleinement à ces deux grands types d'interprétation.

On peut dire que le péril religieux est plus qu'un argument de façade à l'usage des antiféministes, ne serait-ce que parce qu'il est repris par les féministes eux-mêmes. Aussi, à moins d'affirmer que même les féministes étaient misogynes et ne cherchaient qu'à camoufler leur hostilité au suffrage des femmes, on ne peut réduire cet argument à une tactique au service de l'exclusion des femmes. Ce sont au contraire les arguments antiféministes qui viennent justifier la position parfois trop ouvertement électoraliste des sénateurs. En effet, si l'on en juge par la fréquence des expressions de ce type qui relèvent bien souvent de la plus basse misogynie, on remarquera non seulement que

\footnotetext{
${ }^{79}$ Citation célèbre de Renan, reprise par Joseph Barthélémy qui, tout en se déclarant favorable au suffrage des femmes, reconnaît que "dans les premiers temps, un grand nombre d'entre elles obéiraient bien moins à des convictions personnelles qu'à des influences étrangères". Joseph Barthélémy, Le vote des femmes..., op. cit., pp. 484-485.

${ }^{80}$ Cf. Pierre Rosanvallon, Le sacre du citoyen..., op. cit., p. 394.

${ }^{81}$ C'est vrai aussi bien pour l'historiographie américaine que française ; cf. Karen Offen, "Les femmes, la citoyenneté et le droit de vote en France, 1789-1993”, in Yolande Cohen et Françoise Thébaud (éd.), Féminismes et identités nationales. Les processus d'intégration des femmes au politique, Programme Rhône-Alpes de recherche en sciences humaines, 1998, pp. 4770 ; Sian Reynolds, France Between the Wars : Gender and Politics, 1996 ; Christine Vérot, La Question du vote des femmes devant le parlement, Lille III, 1987 ; ainsi que Steven Hause et Anne Kenney, op. cit.

82 Jean-François Sirinelli souligne la distance entre le danger que constituerait l'extrême droite à cette époque (et donc la menace d'une disparition de la République), que l'on peut considérer comme nul ; et le sentiment qu'a la gauche démocratique de détenir la République et de voir dans son propre renversement le début de la fin du régime selon son cœur. Cf. JeanFrançois Sirinelli, "Aux marges de la démocratie. De l'agora à la place des pas perdus", in La démocratie en France, sous la dir. de Marc Sadoun, Paris, Gallimard, 2000, vol. 2, Limites. Claude Langlois, quant à lui, insiste bien sur le fait que l'entredeux-guerres marque le moment où le camp laïc est le mieux structuré, où les affrontements, au moins verbaux, reprennent de plus belle ; pièce de choix, dit-il : Jeanne d'Arc, canonisée en 1920, honorée comme la "sainte de la patrie" par la Chambre bleu horizon qui vote l'instauration d'un jour de fête nationale en son honneur. Cf. Claude Langlois, op. cit. Francine MuelDreyfus, enfin, insiste sur la période des années 1920 qui voient émerger un féminisme catholique de droite qui n'est pas pour rassurer les partis de gauche. N'oublions pas en outre que le pape, en 1919, se déclare favorable au vote des femmes. Cf. Francine Muel-Dreyfus, op. cit.l
} 
celle-ci ne semble pas honteuse mais qu'elle a également toutes les caractéristiques d'une rhétorique destinée à chercher des raisons pour justifier la décision tout à fait politique et opportuniste de ne pas donner le droit de suffrage à un électorat aussi dangereux pour l'avenir de la gauche démocratique : l'hilarité que ces expressions provoquent et l'espèce de légèreté qui en entoure leur réception par l'auditoire, mis en balance avec le sérieux et la passion des débats lorsqu'est abordée la question du comportement électoral présumé des femmes, ne laissent que peu de doute sur la cause du refus des sénateurs. Elles font penser que l'on est sorti du débat au sens strict pour entrer dans une dimension festive destinée surtout à renforcer la communauté de pensée des sénateurs de la gauche démocratique ${ }^{83}$. L'affichage de la misogynie est en outre réparti de manière trop partisane, dans ce débat ${ }^{84}$, pour qu'on n'y voie pas aussi une tactique des sénateurs de la gauche démocratique obéissant au mot d'ordre d'exprimer le plus d'hostilité possible au projet ${ }^{85}$.

La seconde interprétation donne au refus de donner le droit de suffrage aux femmes des raisons de philosophie politique : soit que la doctrine juridique française, de tradition individualiste, ne pouvait intégrer les revendications des femmes en tant que groupe sexué aux intérêts spécifiques ${ }^{86}$; soit au contraire que la doctrine libérale au fondement du droit de suffrage féminin ait été trop individualiste pour les partis français de la IIIème République, surtout préoccupés de catégories telles que la famille, la classe sociale ou le solidarisme ${ }^{87}$.

L'argumentaire déployé dans les chambres de 1919 et 1922 a montré qu'une même idéologie, sexualiste, imprégnait les débats, sans jamais faire référence à l'opposition entre individualisme républicain et revendications féministes. Est-ce à dire que l'idéologie individualiste fonctionne de manière plus diffuse, voire à l'insu des sénateurs ? Comment comprendre dans ce cas l'attitude des députés en 1919 ? Celle-ci reste inexplicable; et elle l'est autant au regard de l'interprétation anglo-saxonne, pour qui les parlementaires français

\footnotetext{
${ }^{83}$ Le président de la Commission n'est pas exempt de ces emportements, qui termine son discours par ce bon mot : "Quand on songe aux conséquences incalculables pour le pays que peuvent avoir les conférences engagées entre diplomates des diverses nations, (...) est-ce bien le moment de faire appel à l'intervention des femmes, pauvres femmes qui oublient qu'elles sont le plus souvent nos maîtresses (Hilarité), et qui aspirent à devenir nos égales ?" Intervention dans la séance du 21 novembre 1922 au Sénat.

${ }^{84}$ On trouve des sénateurs pour reprocher ouvertement aux antiféministes, tel François Labrousse, leur faconde comme l'inanité de leurs arguments ; mais ce sont des sénateurs socialistes ou de droite. Le dialogue qui suit, entre Flaissières (sénateur socialiste) et Labrousse, est aussi savoureux que révélateur du débat :

M. Flaissières. "... Pourquoi [la femme] réfléchit-elle plus que nous ne le faisons, quoi que puisse croire notre excellent collègue M. Labrousse, qui a oublié, peut-être, que l'on ne doit jamais battre une femme, même avec une fleur. (Sourires.) M. Labrousse. Où avez-vous vu, dans mon discours, que je battais les femmes ? (Très bien! à gauche.)

M. Flaissières. A toutes les pages du compte rendu officiel de votre discours". Intervention de Flaissières, dans la séance du 21 novembre 1922 au Sénat.

A droite de l'échiquier politique, Paul d'Estournelles de Constant, prix Nobel de la paix, membre de l'union républicaine, réfute systématiquement les arguments de François Labrousse pour tenter de prouver, par l'exemple américain qu'il connaît bien, que l'inclusion démocratique des femmes n'aurait pas les conséquences désastreuses que "le très savant docteur Labrousse", sur la base de ses seules lectures, veut bien prédire. Voir son intervention dans la séance du 16 novembre 1922. Plus à droite encore, c'est l'avocat Julien Busson-Billault qui mène la charge contre l'antiféminisme de François Labrousse. Voir son intervention dans la même séance.

${ }^{85}$ Suzanne Grinberg, qui fut témoin du succès du docteur Labrousse, a le sentiment que l'auditoire de gauche exprime plus qu'une adhésion à la misogynie de l'orateur en riant ainsi à tout propos : "Devant une assemblée impartiale M. Labrousse n'eut peut-être point fait triompher sa cause. Mais il était avéré que la plupart des membres du sénat avaient un parti-pris et que le groupe radical avait mandat de se montrer hostile." Suzanne Grinberg, Historique du mouvement suffragiste..., op. cit., p. 166.

${ }^{86}$ Cf. Pierre Rosanvallon, Le sacre du citoyen..., op. cit.

${ }^{87}$ Steven Hause et Anne Kenney, Women's suffrage..., op. cit.
} 
font preuve d'un "collectivisme" inapte à intégrer la demande ultraindividualiste des femmes. Mettre la focale sur le vote de 1922 tout en adoptant un point de vue de philosophie politique est périlleux en soi : comment, sur la base d'une idéologie par définition commune à une classe politique donnée (que ce soit celle au pouvoir ou celle des parlementaires dans leur ensemble), comprendre deux votes contradictoires, sinon en réduisant l'un des deux au rang de "cynisme" ou d'accident de l'histoire ? La cohérence ainsi retrouvée reste problématique : car c'est une cohérence externe, adaptée a posteriori aux catégories d'une idéologie qu'on postule et dont on ne peut vérifier l'existence dans des débats qui n'en montrent pas trace. Tout au contraire, ceux-ci révèlent une adhésion massive, des partisans comme des opposants, de la gauche comme de la droite, à l'idéologie sexualiste. Cette idéologie sexualiste n'est pour autant pas en cause, dans l'issue négative du débat de 1922 et c'est au-delà de l'explication philosophique qu'il faut chercher les éléments explicatifs du vote des deux assemblées. On l'a vu, ce sont les conséquences attendues de la réforme qui obligent les sénateurs à ne pas céder aux sirènes de la métaphysique, trop légèrement mise en loi, selon eux, par les députés. Leur devoir de responsabilité, historiquement attribué, se double ici d'un pragmatisme très conjoncturel imposé par des circonstances tout à fait particulières ; aux députés qu'ils soupçonnent d'hypocrisie, les sénateurs répondent par le réalisme et la juste mesure des conséquences de leur acte ; leur position est simple, forte, sans état d'âme, parce qu'elle n'est pas (plus) une question de décider si les femmes ont droit au vote, mais de savoir, par expérience personnelle ou par adhésion à une évidence de la libre-pensée devenue lieu commun de tous les parlementaires, que les femmes ne pourront pas voter correctement, ni en terme de comportement (liberté de conscience) ni en terme de résultat (vote républicain). Aussi, les contradictions qui nuisent au suffrage des femmes sont-elles moins entre les universalistes et les féministes, ou entre les féministes universalistes et les féministes "corporatistes", qu'entre le principe dont la bonté est à peu près admise par tous, y compris par la droite du Sénat en 1922, et l'opportunité de son application ; c'est-à-dire dans la distance entre le pouvoir d'énonciation symbolique des uns et le souci de pragmatisme des autres.

S'il faut "tirer un peu plus haut" l'explication, pour dépasser les enjeux de pouvoir et considérer les motivations idéologiques, on peut s'intéresser à ce qui, par définition, est commun aux parlementaires. Expliquer le vote de 1922 par la position institutionnelle ne dispense pas en effet d'observer le rôle de l'idéologie sexualiste qui a conduit les uns à redouter l'homogénéité et la spécificité du comportement électoral des femmes et les autres à en faire au contraire un argument en faveur du besoin féminin de représentation politique. Mais de toute évidence, si l'idéologie oriente le débat, elle n'augure pas de son issue ; et de 1919 à 1922, celle-ci varie au gré de convictions qui dépendent du degré d'implication des parlementaires dans le processus législatif, c'est-à-dire de leur possibilité de faire plus ou moins fi des conséquences attendues du postulat sexualiste qu'ils partagent tous. En 1922, c'est cette vision sexualiste du corps électoral qui donne tout son

\footnotetext{
${ }^{88}$ On l'aura peut-être remarqué par défaut : la contradiction apparaît peu dans les débats qui ne se placent jamais à ce niveau théorique mais cherchent au contraire à s'en dégager au maximum pour mieux prouver leur légitimité, y compris de la part des féministes comme Louis Martin.
} 
poids aux considérations de tactique en déterminant les sénateurs à repousser l'extension démocratique du suffrage au nom de la sauvegarde de l'ordre républicain. Si singularité française il y a, elle tient en partie à cette coïncidence entre des représentations globalement sexualistes du corps électoral, le sentiment de la gauche démocratique d'une fragilisation de son pouvoir et, enfin, la situation temporelle et institutionnelle des sénateurs qui contraint ceux-ci, après le vote de justice et de principe de la Chambre, à faire le choix du pragmatisme $^{89}$.

81694 car.

Notice biographique :

Anne Verjus, politiste, est chercheur au CNRS (Groupe d'Analyse des Politiques Publiques-ENS de Cachan). Elle travaille principalement sur l'évolution des représentations, que ce soit dans le champ historique de la citoyenneté (notamment au travers de la situation politique des femmes et de la famille depuis 1789) ou dans celui, plus contemporain, des politiques de la division des sexes. Elle a publié plusieurs articles sur ces questions (notamment "Vote familialiste et vote familial. Contribution à l'étude du processus d'individualisation des femmes dans la première partie du XIXème siècle, in Genèses, $\mathrm{n}^{\circ} 31$, juin 1998) et prépare actuellement la publication de sa thèse.

\footnotetext{
${ }^{89}$ Je dois tous mes remerciements à V. Antomarchi, V. Bussat, F. Caille, P. Fache, P. Lascoumes, M. Ozouf et A. Tonnelé, lecteurs attentifs dont les conseils ont contribué à la réalisation de cet article.
} 